Artigo / Article

\title{
Programa de Triagem Neonatal para Hemoglobinopatias em Dourados, MS - uma análise
}

\author{
Neonatal Screening Program for Hemoglobinopathies in Dourados, MS - an analysis
}

Renata A. Volpe de Souza ${ }^{1}$

Riccardo Pratesi $i^{2}$

Silvana F. Fonseca ${ }^{3}$
Embora estejam bem definidos os beneficios da implantação do programa de triagem neonatal para hemoglobinopatias, não são raros os estudos que apontam falhas nesses programas. Este estudo teve como objetivo avaliar o programa de triagem neonatal para hemoglobinopatias no municipio de Dourados, estado do Mato Grosso do Sul (MS). Foram entrevistadas, através da aplicação de formulários, 32 famílias cujos filhos foram identificados como portadores de hemoglobinopatias, durante a triagem neonatal, no periodo de janeiro de 2000 a dezembro de 2005. Adicionalmente, foram verificadas a cobertura do Programa Nacional de Triagem Neonatal (PNTN) e a incidência de hemoglobinopatias no MS, de 2000 a 2005. Dos 242 casos de hemoglobinopatias diagnosticados neste periodo, a heterozigose para hemoglobina $S$ demonstrou incidência de 1,37\%, a heterozigose para hemoglobina C, 0,37\% e a heterozigose para hemoglobina $D, 0,007 \%$. Não foram diagnosticados casos de anemia falciforme. A cobertura encontrada foi de $81,4 \%$. Foram detectadas falhas, como a não reconvocação para o exame confirmatório, ausência de encaminhamento médico para orientação, falta de investigação familiar e a falha na compreensão do aconselhamento genético. Rev. Bras. Hematol. Hemoter. 2010;32(2):126-130.

Palavras-chave: Triagem neonatal; hemoglobinopatias; avaliação de programa.

\section{Introdução}

O desenvolvimento socioeconômico, o controle dos fatores ambientais e os grandes avanços científicos fizeram com que as patologias infecciosas deixassem de ser o principal e único problema de saúde pública. Nesse contexto, de transição epidemiológica, surgiram novos desafios para os profissionais que lidam com a saúde pública, cujo enfoque voltase também para a prevenção e controle das doenças genéticas. Dentre as patologias genéticas mais prevalentes em todo o mundo, as hemoglobinopatias ocupam um lugar de destaque. Cerca de 270 milhões de pessoas são portadores de hemoglobinas anormais em diferentes combinações e quadros clínicos, que variam de assintomáticos a letais. ${ }^{4,16}$ No Brasil, devido à grande miscigenação racial entre os povos colonizadores, houve dispersão de genes, principalmente aqueles relacionados às síndromes falciformes e talassêmicas. ${ }^{10}$

Para a prevenção e controle das hemoglobinopatias, alguns programas de saúde foram implementados no Brasil. Dentre estes, destaca-se a Portaria $n^{\circ} 822$ do Ministério da Saúde, que instituiu, no âmbito do Sistema Único de Saúde, o Programa Nacional de Triagem Neonatal (PNTN), garantindo a todos os recém-nascidos brasileiros igual acesso aos testes de triagem. O PNTN visa o diagnóstico precoce e a orientação terapêutica de anormalidades no metabolismo do recém-nascido, bem como a orientação necessária aos pais. ${ }^{12}$

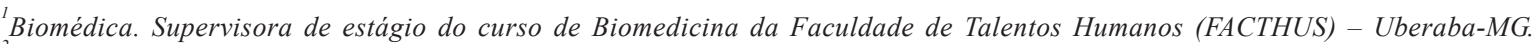
${ }_{3}^{2}$ Médico pediatra. Professor do Programa de Pós-graduação da Faculdade de Medicina da Universidade de Brasília (UnB), Brasilia, DF. ${ }^{3}$ Médica hematologista pediátrica. Professor-orientador do Programa de Pós-graduação em Ciências da Saúde da Faculdade de Ciências da Saúde - UnB, Brasília, DF.
}

Universidade de Brasília (UNB) - Distrito Federal.

Correspondência: Silvana Fahel da Fonseca

SQN 214, bloco C, ap. 214, Asa Norte

70873br-030 - Brasília-DF - Brasil

E-mail:sfahel@unb.br

Doi: 10.1590/S1516-84842010005000037 
Na literatura científica existem poucos trabalhos que avaliam o Programa de Triagem Neonatal e, embora estejam claros os seus benefícios para o diagnóstico precoce das hemoglobinopatias e orientação das famílias de neonatos portadores de tais patologias, não são raros os estudos que apontam falhas nesses programas. ${ }^{2,8,17,19,20,22}$ Como apropriadamente discutido por Meirelles, ${ }^{11}$ as análises críticas são necessárias e benéficas e possibilitam modificações que favorecem o melhor desempenho desses programas. Assim, frente à alta morbidade das hemoglobinopatias e do grande impacto que o diagnóstico precoce exerce nas taxas de morbi-mortalidade das mesmas, o presente estudo objetiva avaliar a efetividade do Programa de Triagem Neonatal para hemoglobinopatias no município de Dourados, MS, e verificar a incidência de hemoglobinopatias nesta população.

\section{Casuística e Método}

O projeto foi aprovado pelo Comitê de Ética em Pesquisa (CEP) do Centro Universitário da Grande Dourados e seguiu as normas de ética em pesquisa.

Trata-se de um estudo descritivo do tipo ambispectivo, no qual, na vertente retrospectiva, foram analisados os dados de arquivo do PNTN do município de Dourados, MS, no período de 2001 a 2005, e, na vertente prospectiva, foi aplicado um formulário às famílias de recém-nascidos com hemoglobinopatias, identificadas pelo programa de triagem neonatal, após a assinatura do termo de consentimento livre e esclarecido. A escolha deste município deve-se ao fato dessa cidade ter a segunda maior população do estado do MS e participar deste Programa desde a sua implantação pelo governo federal.

As famílias foram selecionadas de forma aleatória, através de sorteio, visando compor uma amostra de 32 pais ou responsáveis pelos recém-nascidos, cujo número foi previamente calculado através do programa Epi Info versão 3.3.2, com margem de erro de $2 \%$ e nível de significância de $99 \%$. Foram incluídos todos os pais ou responsáveis sorteados que concordaram em participar da pesquisa e excluídos os não encontrados e a população indígena.

O grau de compreensão sobre o aconselhamento genético oferecido pelo Programa de Triagem Neonatal às famílias com crianças portadoras de traço falciforme foi avaliado através de questões abertas e fechadas.

As respostas foram classificadas em compreensão satisfatória e compreensão insatisfatória após verificar se estas se enquadravam ou não nos requisitos contidos na definição de Aconselhamento Genético (AG) adotada pela Sociedade Americana de Genética Humana. ${ }^{?}$

Os dados foram tabulados e expressos por meio de ferramentas estatísticas através do programa Excel 7.0 e para cálculo da cobertura do programa utilizou-se a fórmula: cobertura $=$ número de rastreados $\mathrm{x}$ 100/ número de nascidos vivos. As análises estatísticas dos dados basearam-se em cálculos de incidência das variáveis quantitativas e em algumas realizou-se a comparação de incidências pelo teste do qui - quadrado $\left(\chi^{2}\right)$, considerandose $5 \%$ como nível de significância.

\section{Resultados}

Na Tabela 1 pode ser observado o crescente número de recém-natos participantes do PNTN no município de Dourados que, no ano de 2005, apresentou $82 \%$ de cobertura.

A incidência de hemoglobinas anômalas nesta população, no período de 2001 a 2005, foi de $1,75 \%$. Dos três tipos de heterozigoses detectados, o traço falciforme foi o mais incidente, ocorrendo em $1,37 \%$ dos recém-nascidos triados. Não foram identificados casos de doença falciforme (Tabela 2).

Todas as 32 famílias sorteadas para aplicação do formulário de avaliação do acompanhamento eram de crianças com traço falciforme. Destes, $12(37,5 \%)$ afirmaram que os recém-nascidos não foram convocados para repetição de exame e confirmação diagnóstica. As vinte famílias que receberam a solicitação de repetição do teste de triagem realizaram a segunda coleta, sendo os resultados confirmados.

\begin{tabular}{|c|c|c|c|}
\hline Ano & $\begin{array}{c}\text { População } \\
\text { triada }\end{array}$ & $\begin{array}{c}\text { Nascidos } \\
\text { vivos }\end{array}$ & Cobertura (\%) \\
\hline 2001 & 2.466 & 3.614 & 68,2 \\
\hline 2002 & 2.622 & 3.282 & 79,9 \\
\hline 2003 & 2.793 & 3.560 & 78,5 \\
\hline 2004 & 3.025 & 3.700 & 81,8 \\
\hline 2005 & 2.929 & 3.573 & 82,0 \\
\hline Total & 13.835 & 17.729 & 81,4 \\
\hline
\end{tabular}

Tabela 2. Incidência de hemoglobinas anômalas detectadas pelo Programa Nacional de Triagem Neonatal no município de Dourados- MS, (janeiro/2001 a dezembro/ 2005)

\begin{tabular}{cccccccc}
\hline Ano & $\begin{array}{c}\text { População } \\
\text { triada }\end{array}$ & \multicolumn{2}{c}{ Hb AS } & \multicolumn{2}{c}{ Hb AC } & \multicolumn{2}{c}{ Hb AD } \\
\hline 2001 & 2.466 & 27 & 1,09 & 6 & 0,24 & 1 & 0,04 \\
2002 & 2.622 & 28 & 1,06 & 10 & 0,38 & 0 & 0 \\
2003 & 2.793 & 41 & 1,47 & 7 & 0,25 & 0 & 0 \\
2004 & 3.025 & 49 & 1,62 & 16 & 0,52 & 0 & 0 \\
2005 & 2.929 & 45 & 1,53 & 12 & 0,4 & 0 & 0 \\
\hline Total & 13.835 & 190 & 1,37 & 51 & 0,37 & 1 & 0,007 \\
\hline
\end{tabular}


Os resultados sobre a investigação da hemoglobinopatia nos pais de recém-nascidos afetados são demonstrados na Tabela 3. Vinte e cinco, dos 32 casais entrevistados, receberam a orientação para realização de exame para investigação de hemoglobinopatias. Ocorreram quatro solicitações de exames só para a mãe e três casais afirmaram não terem sido solicitados (Tabela 3 ).

Das 32 famílias entrevistadas, 29 receberam orientações sobre a alteração encontrada no exame dos seus filhos. Doze, destas 29 famílias, não foram solicitadas a repetir o exame dos neonatos, ou seja, foram orientadas com o exame inicial, de triagem.

Menos de 50\% das famílias entrevistadas demonstrou compreensão satisfatória dos dados de aconselhamento genético e conhecimento sobre a heterozigose. A maioria das pessoas com compreensão insatisfatória não apresentava qualquer grau de escolaridade (Tabela 4).

Tabela 3. Frequência dos indivíduos que realizaram o exame para detecção de hemoglobinopatias entre os solicitados

\begin{tabular}{ccccc}
\hline & \multicolumn{2}{c}{ Exame solicitado } & \multicolumn{2}{c}{ Fez exame } \\
\hline & $\mathrm{n}$ & $\%$ & $\mathrm{~N}$ & $\%$ \\
\hline Mãe & 29 & 90,6 & 27 & 93,1 \\
Pai & 25 & 78,1 & 18 & 72,0 \\
Total & 54 & & 45 & \\
\hline
\end{tabular}

$p=0,031 ; \chi^{2}=4,63$

Tabela 4. Relação entre o grau de escolaridade dos pais e a compreensão ao aconselhamento genético

\begin{tabular}{ccccc}
\hline Escolaridade & \multicolumn{2}{c}{ C. Satisfatória } & \multicolumn{2}{c}{ C. Insatisfatória } \\
\hline & $\mathrm{n}$ & $\%$ & $\mathrm{~N}$ & $\%$ \\
\hline Analfabeto & 0 & 0,0 & 13 & 86,7 \\
E. fundamental & 11 & 78,6 & 2 & 13,3 \\
E. médio & 3 & 21,4 & 0 & 0,0 \\
Total & 14 & 100,0 & 15 & 100,0 \\
\hline $\mathrm{p}=0,000159 ; \chi^{2}=18,63$ & & &
\end{tabular}

\section{Discussão}

De 2001 a 2005, no município de Dourados, MS, foram detectados, durante a triagem neonatal, 242 casos de heterozigoses para hemoglobinopatias, sendo que o traço falciforme demonstrou incidência de $1,37 \%$, contra $0,37 \%$ para hemoglobinopatia C e $0,007 \%$ para hemoglobinopatia D. A incidência do traço falciforme neste estudo foi menor que a relatada no Rio de Janeiro (3,96\%), ${ }^{10}$ no estado de São Paulo $(2,0 \%)^{5}$ e no Rio Grande do Norte $(1,5 \%)^{3}$, e a incidência da heterozigose para $\mathrm{Hb} \mathrm{C}$ foi menor que a encontrada no Rio de Janeiro e São Paulo, semelhante àquela encontrada no Rio Grande do Norte $(0,31 \%)^{3}$ e maior que a referida no Rio Grande do Sul, $(0,19 \%) .{ }^{21}$ A incidência dos tipos de hemoglobinopatia é influenciada pela origem racial da população, e a diferença de incidência encontrada nas regiões brasileiras reflete a formação racial nestes grupos populacionais.

É interessante ressaltar a ausência de casos de homozigoses para a $\mathrm{HbS}$ e para a beta talassemia durante os cinco anos estudados. Considerando-se que a região de Dourados foi colonizada por povos de diversas origens raciais e que sofreu intensa influência de migrações gaúchas, ${ }^{9}$ esperavase a identificação de casos de beta talassemia no município. Uma provável explicação para o resultado encontrado pode ser a impossibilidade de identificação, sem a utilização de técnicas de biologia molecular, da homozigose para mutações $\beta+$ talassêmicas e da heterozigose para a $\beta$-talassemia, no período neonatal.

Uma das intenções deste programa é alcançar a cobertura de $100 \%$. No presente estudo verificou-se que embora o programa ainda não tenha atingido esta meta, a cobertura vem aumentando ao longo dos anos, com média de $81,3 \%$ nos cinco anos analisados. Trabalhos semelhantes em diversas partes do País têm demonstrado taxas de coberturas variáveis, de $32,2 \%$ a $100 \%$. $^{2,6,18,19,23}$

No estado do MS foram encontradas coberturas de $80,4 \%$ no município de Cassilândia, durante o período de 2001 a 2005, ${ }^{22}$ e $89,3 \%$ como média geral do estado do Mato Grosso do Sul, no ano de $2002 .{ }^{6}$

Ao se comparar a cobertura encontrada neste estudo com resultados de outras localidades, verifica-se que há uma variação visível entre as regiões brasileiras, o que pode ser explicado pela heterogeneidade de estrutura da rede de atendimento do PTN e do perfil cultural e socioeconômico da população, o que é bem elucidado em pesquisa realizada no recôncavo baiano, a qual observou coberturas totalmente diferentes em três municípios do mesmo estado no ano de 2003, sendo 51,6\% para Cachoeira, 40,0\% para Maragogipe e $100 \%$ para São Fêlix. ${ }^{19}$

Se nos apoiarmos, nos relatos de Oliveiri e colaboradores, ${ }^{15}$ que afirmam que em países tecnologicamente avançados, como a Itália, foram necessários 17 anos para atingir uma cobertura de $100 \%$ no PTN, e se considerarmos o fato de que os dados deste estudo podem estar subestimados, uma vez que não foram analisados os dados da rede privada, julgamos como satisfatório o resultado da cobertura do PNTN no município de Dourados, a qual vem aumentando ao longo do tempo e está acima do nível mínimo exigido na Portaria $\mathrm{n}^{\circ}$ 822 do Ministério da Saúde, a qual estabelece a cobertura maior ou igual a $70 \%$ como necessária para se habilitar para a fase III do programa. ${ }^{12}$

O acompanhamento dos casos positivos de hemoglobinopatias permitiu verificar que, entre os 32 neonatos que participaram da pesquisa, $12(37,5 \%)$ não foram convocados para a repetição do teste e não realizaram o exame confirmatório. Este dado é preocupante, pois, segundo Orlando et al. ${ }^{16}$ os pontos fundamentais de um programa preventivo de hemoglobinopatias compreendem a divulgação da informação à população, o reconhecimento de heterozigotos, diagnóstico 
neonatal e aconselhamento genético, os quais são precedidos pelo aviso sobre a alteração do teste e a convocação para a realização do exame confirmatório.

Ao se verificar a solicitação e realização de exames diagnósticos para hemoglobinopatias, em pais de neonatos triados, evidenciou-se que a adesão das mães que haviam sido convocadas para realizar o teste foi significativamente maior que a dos pais $\left(\mathrm{p}=0,031 ; \chi^{2}=4,63\right)$. Das 29 mães convocadas, $27(93,1 \%)$ o fizeram e, entre o total de 25 pais, $18(72,0 \%)$ fizeram o exame. Um casal relatou não ter feito o teste por desinteresse.

Estes resultados demonstram que, durante o acompanhamento do recém-nascido afetado, a avaliação do perfil fenotípico dos casais não está sendo realizada, podendo isto dever-se à não solicitação do exame e/ou não adesão à solicitação por parte do casal. Sugere-se ainda que as mães estiveram mais comprometidas com o cuidado da saúde familiar, como também mais disponíveis, ou por estarem em licença-maternidade ou responsáveis apenas pelos cuidados domésticos, e assim com maior disponibilidade de tempo para a coleta do exame. Isso aponta a necessidade de uma maior atenção e customização por parte dos profissionais envolvidos com o acompanhamento destas famílias, a fim de realizar exames de todos os casais cujos filhos são portadores de hemoglobinopatias e criar formas de atuação que também atinjam os pais, que, na maioria das vezes, se encontram mais empenhados no trabalho para sustento da família, deixando para a mãe este tipo de responsabilidade.

A Portaria $\mathrm{n}^{\circ}$ 1391/GM, de 16 de agosto de 2005, preconiza a instituição de uma política de capacitação de todos aqueles envolvidos, que promova a educação permanente e a promoção do acesso à informação e ao aconselhamento genético aos familiares e às pessoas com a doença ou o traço falciforme.

A possibilidade de realização de exames nos demais membros da família também é considerada um grande benefício da triagem neonatal para hemoglobinopatias por outros autores, ${ }^{10,14,21}$ principalmente devido à detecção de possíveis portadores de traço falciforme, que é o fenótipo mais encontrado no Brasil, assim como a identificação de pessoas com doença falciforme com apresentação mais benigna. Isso levaria a posterior orientação quanto ao risco genético, pois, embora sejam saudáveis, podem gerar crianças com a anemia falciforme.

Uma vez que todos os participantes que responderam o formulário eram pais de crianças com traço falciforme, e a compreensão do aconselhamento genético nestes casos é o ponto principal do acompanhamento, deve-se considerar que o PNTN não alcançou o seu objetivo nesta população, não foi eficaz, uma vez que mais da metade $(51,7 \%)$ não compreendeu totalmente os esclarecimentos dados pelo clínico.

Silva e Yamaguchi consideram que muitos clínicos brasileiros ainda percebem o aconselhamento genético dos pacientes ou de seus pais como um procedimento secundário, opcional ou de responsabilidade exclusiva do geneticista. Advertem que a possibilidade de uma alteração genética ter caráter hereditário acrescenta componentes adicionais à conduta clínica e à responsabilidade médica, e que o aconselhamento genético é um componente importante da conduta médica, sendo sua omissão uma falha grave. As pessoas devem ser conscientizadas do problema, sem serem privadas do seu direito de escolha. ${ }^{20}$

A tentativa de correlacionar os graus de compreensão com a escolaridade demonstrou que a grande maioria das pessoas que apresentaram compreensão insatisfatória não tinha qualquer escolaridade $(86,7 \%)$ ou possuia apenas o ensino fundamental. Entre os que compreenderam as informações recebidas, $78,6 \%$ cursaram ensino fundamental, e o restante $(21,4 \%)$, ensino médio, não existindo pessoas não alfabetizada entre eles. Estes resultados revelam que a compreensão foi significativamente menor nos indivíduos com pouco grau de escolaridade e nos permite concluir que, embora uma grande parte da responsabilidade sobre a eficiência e eficácia do Programa de Triagem Neonatal recaia sobre os profissionais envolvidos com este trabalho, fica evidente que não somente a orientação dos familiares, mas também a educação da população é ainda um grande problema brasileiro que reflete no resultado de qualquer programa, dificultando o alcance de seus objetivos. Assim, para solucionar este problema seriam necessárias intervenções mais abrangentes no campo educacional, as quais estão diretamente ligadas a fatores socioeconômicos, culturais e pisicológicos da população.

Os dados obtidos neste estudo sugerem que a análise de resultados de um programas de saúde, como o PNTN, deve transpor as taxas de cobertura alcançada e acompanhar a população alvo a curto, médio e longo prazos, verificando o impacto que as medidas implantadas causaram.

\footnotetext{
Abstract

Although the benefits of implanting a neonatal screening program for hemoglobinopathies are well defined, there are many studies that point to failures in these programs. The objective of this study was to evaluate the efficiency of the neonatal screening program for hemoglobinopathies in the municipality of Dourados, in the state of Mato Grosso do Sul, Brazil. Between January 2000 and December 2005, thirty-two families with children diagnosed during neonatal screening as having hemoglobinopathies were evaluated using questionnaires. Additionally, the coverage of the Brazilian National Neonatal Screening Program and the incidence rates of hemoglobinopathies in the state of Mato Grosso do Sul between 2000 and 2005 were analyzed. Of the 242 cases of hemoglobinopathies that were diagnosed in this period, $1.37 \%$ individuals were identified as heterozygotes for hemoglobin S, $0.37 \%$ for hemoglobin C, and $0.07 \%$ for hemoglobin D. There were no cases of sickle cell anemia diagnosed during this period. The program was found to be positive and provided a broad coverage of $81.4 \%$.
} 
Some failures were detected such as not requiring patients to return for confirmation examinations, the lack of medical counseling and follow through, lack of investigations of family histories, and lack of understanding on the part of the family of the genetic counseling they received. Rev. Bras. Hematol. Hemoter. 2010;32(2):126-130.

Key words: Neonatal screening; hemoglobinopathies; program evaluation.

\section{Referências Bibliográficas}

1. Adorno EV, Couto FD, Moura Neto JP, Menezes JF, Rêgo M, Reis MG, et al. Hemoglobinopatias em recém-nascidos de Salvador, Bahia, Nordeste do Brasil. Cad Saude Publica. 2005;21(1):292-8.

2. Almeida AM, Godinho TM, Teles MS, Rehem AP, Jalil HM, Fukuda TG, et al. Avaliação do Programa de Triagem Neonatal na Bahia no ano de 2003. Rev Bras Saude Mater Infant. 2006; 6(1):85-91.

3. Araújo CPE, Serafim EDS, Castro Júnior WAP, Medeiros, TMD. Prevalência de hemoglobinas anormais em recém-nascidos da cidade de Natal, Rio Grande do Norte, Brasil. Cad Saude Publica. 2004;20 (1):123-8.

4. Backes CE, Mallmann TD, Bazzo ML, Santos-Silva MC. Triagem neonatal como um problema de saúde pública. Rev Bras Hematol Hemoter. 2005;27(1):43-7.

5. Brandelise S, Pinheiro V, Gabetta CS, Hambleton I, Serjeant B, Serjeant G. Newborn screening for sickle cell disease in Brazil: the Campinas experience. Clin Lab Haematol. 2004;26(1):15-9.

6. Carvalho TM, Vargas PR, Santos HMGP. Triagem neonatal: um programa Nacional de Saúde Pública. Triagem. 2002;4:5-6.

7. Epstein CJ. Genetic counseling: statement of the American Society of Human Genetics ad hoc Comittee on Genetic Counseling. American Journal of Human Genetics 1975;27(2):241-2.

8. Farrell M, Certain L, Farrell P. Genetic counseling and risk communication services of newborn screening programs [abstract]. Arch Pediatr Adolesc Med. 2001;155(2)120-6.

9. Gressler LA, Swensson LJ. Aspectos históricos do povoamento e da colonização do estado do Mato Grosso do Sul. São Paulo: Câmara do livro; 1988.

10. Lobo CLC, Bueno LM, Moura P, Ogeda LL, Castilho S, Carvalho SMF. Triagem neonatal para hemoglobinopatias no Rio de Janeiro, Brasil. Rev Pan Salud Publica. 2003;13(2):154-9.

11. Meirelles RMR. Triagem neonatal: ficção ou realidade? Arq Bras Endocrinol Metab. 2000;44(2):119-20.

12. Ministério da Saúde (MS). Portaria GM/MS n. 822/ GM em 6 de junho de 2001. Instituição do Programa Nacional de triagem neonatal, no âmbito do XX. Sistema Único de Saúde, para Fenilcetonúria, Hipotireoidismo Congênito, Fibrose Cística e Hemoglobinopatias - Brasília, MS/ jun. 2001.

13. Ministério da Saúde (MS). Portaria n. 1.391/ GM em 16 de agosto de 2005. Institui no âmbito do Sistema Único de Saúde, as diretrizes para a Política Nacional de atenção Integral às Pessoas com Doença Falciforme e outras Hemoglobinopatias. - Brasília, MS/ ago. 2005.

14. Modell B. Etica del diagnóstico prenatal y asesoramiento genético. Foro Mund Salud 1990;11:179-86.

15. Oliveiri A, Fazzini C, Grandolfo ME, Medda E, Stazi MA, D Archivio $\mathrm{M}$, et al. La rivalutazione della diagnosi nelipotiroidismo congenito. Ann Ist Super Sanita. 1999;35(2):273-82.

16. Orlando GM, Naoum PC, Siqueira FAM, Bonini-Domingos CR. Laboratory diagnosis of hemoglobinopathies in different population groups. Rev Bras Hematol Hemoter. 2000;22(2):111-21.
17. Ramalho AS, Magna LA, Silva RBP. Government Directive MS \# 822/01: unique aspects of hemoglobinopathies for public health in Brazil. Cad Saude Publica. 2003;19(4):1195-9.

18. Ramalho RJR, Ramalho ARO, Oliveira CRP, Aguiar-Oliveira MH. Evolution of the screening program for congenital hypothyroidism and phenylketonuria in Sergipe State from 1995 to 2003. Arq Bras Endocrinol Metab. 2004;48(6):890-6.

19. Silva SW, Lastra A, Oliveira SF, Guimarães NK, Grisolia CK. Avaliação da cobertura do programa de triagem neonatal de hemoglobinopatias em populações de Recôncavo Baiano, Brasil. Cad Saude Publica. 2006;22(12):2561-66.

20. Silva, KR, Yamaguchi MU. Os benefícios da inclusão das hemoglobinopatias na triagem neonatal. Arq. Ciênc. Saúde Unipar, Umuarama, 2007;11(1):67-73.

21. Sommer CK, Goldbeck AS, Wagner SC, Castro SM. Neonatal screening for hemoglobinopathies: a one-year experience in the public health system in Rio Grande do Sul State, Brazil. Cad Saude Publica. 2006;22(8):1709-14.

22. Sousa-Polezzi, RC. Distúrbios genéticos detectados através de triagem neonatal no município de Cassilândia-MS. Revista Visão Universitária. 2005;15:53-8.

23. Stranieri I. Avaliação da implantação do Programa de triagem neonatal para hipotireoidismo congênito e fenilcetonúria entre usuários da rede pública. Estado de Mato Grosso, 2003-2004 [dissertação]. Cuiabá (MT): Universidade Federal de Mato Grosso; 2007.

Avaliação: Editor e dois revisores externos

Conflito de interesse: sem conflito de interesse

Recebido: 21/07/2009

Aceito: 16/10/2009 